\title{
New didactic formulation of the laws of reflection of light
}

\author{
Nova formulação didática das leis da reflexão da luz
}

\author{
Ángel Luis Pérez-Rodríguez ${ }^{1}$, Guadalupe Martínez-Borreguero*2, María Isabel Suero-López ${ }^{1}$ \\ ${ }^{1}$ Department of Physics, Faculty of Sciences, University of Extremadura, Badajoz, Extremadura, España \\ ${ }^{2}$ Department of Science and Mathematics Education, University of Extremadura, Badajoz, Extremadura, España
}

\begin{abstract}
Received on November 17, 2016. Revised on December 01, 2016. Accepted on December 07, 2016.
\end{abstract}
\begin{abstract}
This work presents a new formulation of the laws of reflection of light, and it is shown the equivalence between the new and the classic formulations. The proposed formulation has a significant educational value, as it allows drawing analogies between the phenomena of light reflection and elastic collisions, which are very well known by students. The new developed statement of the law of reflection allows the resolution of traditional problems of geometrical optics, and in some cases the solution is much simpler and more intuitive than that obtained using the traditional statement of the laws of reflection. As an example, this formulation is applied for the resolution of the classic reflection problem of the three plane mirrors forming a trirectangular trihedron, also known as corner reflector.
\end{abstract}

Keywords: Reflection of light, laws of reflection, new formulation, vectors, education.

Este trabalho apresenta uma nova formulação das leis de reflexão da luz, mostrando a equivalência com a formulação clássica. A formulação proposta tem um valor educativo bem significativo, pois permite estabelecer analogias entre os fenômenos de reflexão da luz e as colisões elásticas, que são bem conhecidas pelos alunos. O novo enunciado da lei da reflexão permite a resolução de problemas tradicionais da óptica geométrica; em alguns casos, a nova solução é muito mais simples e intuitiva do que a solução obtida através do enunciado tradicional das leis da reflexão. Como exemplo, esta formulação é aplicada para a resolução do problema de reflexão clássico dos três espelhos planos que formam um triedro trirretangular, também conhecido como refletor de canto.

Palavras-chave: Reflexão da luz, leis da reflexão, vetores.

\section{Introduction}

Reflection laws are well known for long and are stated in many classic optics books $[1,2,3,4,5,6$, $7]$. The traditional statement that appears in most textbooks, for example in [5] is divided into two expressions. The first part of the law of reflection, which first appeared in the book catoptrics, whose authorship is attributed to Euclid, is expressed as "the angle of incidence equals the angle of reflection". The second part of the law of reflection states, "The incident ray, the normal to the surface and the reflected ray, lie all in the same plane, called the plane of incidence." This account is similarly stated in other traditional books $[1,2,3,4,6,7]$.

*Endereço de correspondência: mmarbor@unex.es
Despite being an issue considered as settled, in recent years several papers have been published in various journals with new formulations for the laws of reflection. For example, [8] refers to "the generalized vector laws of reflection and refraction". In this paper, the authors points out an ambiguity in the traditional and widely used laws of reflection. As a new feature, they propose the inclusion of a more generalized form for the definition of the angle of incidence and angle of reflection, incorporating the fundamental definition of angle in geometry.

In another recent paper [9] the same authors continue the earlier work on the generalized laws of reflection. This work relates the laws of reflection with the principle of conservation of momentum and makes use of the photon theory conceived by Einstein, given its importance from an analytical academic standpoint. 
Later, in [10] the authors apply their new set of generalized laws of reflection on problems of optical rays rotation. In this paper, a problem on rotating optical mirrors is solved as proof of the usefulness of the laws set forth by the authors.

In another more recent work [11], the same authors apply their new set of generalized vector laws of reflection and make an extensive analysis of their use on spherical surfaces, considering different imaging cases. These authors have observed that direct application of its new set of generalized vector law of reflection leads to the traditional ray optics formulation for each of the cases considered. The analysis of the results made by the authors indicates that, based on these laws, you can obtain the general expressions of the traditional generalized formulation for mirrors.

Recently [12], other authors have point out that it is convenient to use the dot product, in addition to the cross product, when expressing the vector laws of reflection. They suggest that it is possible to modify the vector laws of reflection to incorporate both the cross and the dot product. This is very efficient from a computing point of view, since in this manner wave vectors of the incident and the reflected wave and the normal to the surface are perfectly defined.

Later, paper [13] addresses the issues and questions raised in [12] on generalized vector laws of reflection, establishing a response to that paper. This study argues that the introduction of the dot product is redundant and confirms the validity of their statements of the generalized vector laws of reflection established in $[8,9,10,11]$.

Again, in paper [14], the authors use their statements of the new generalized laws of reflection to arrive at a theoretical proof of Fermat's principle. In this paper, the authors have managed to deduce the Fermat principle from their vector laws of reflection, which proves the efficiency of the generalized vectorial laws of reflection and refraction [8].

As a further step forward, the generalized vectorial laws of reflection and refraction have been applied in a new paper [15] to produce the generalized vectorial laws of multiple reflection and refraction.

In line with this recent work, the study presented here proposes the use of a new formulation of the laws of reflection useful from an academic point of view in the field of optics, and it shows the equiva- lence of this new statement with the classic statement.

\section{New formulation of the laws of reflection of light}

\subsection{Statement}

The statement proposed in this work is as follows:

If at one point on a surface whose orientation in space (or of the tangent plane to the surface, if it is not a plane itself) is defined by a unit vector $\mathbf{k}$ (perpendicular to the surface), strikes an incident ray corresponding to a plane wave (propagating through a homogeneous and isotropic medium) whose direction of propagation coincides with that from a unit vector $\mathbf{u}_{i}$ [expressed in terms of its components with respect to an orthonormal coordinate system, with one of its axis coinciding with the direction of $\mathbf{k}$ $\left.\left(u_{i x} \mathbf{i}+u_{i y} \mathbf{j}+u_{i z} \mathbf{k}\right)\right]$, it will be reflected so that the unit vector whose direction coincides with that from the reflected ray, $\mathbf{u}_{\mathrm{r}}$, will only differ from the unit vector whose direction coincides with that from the incident ray, in the change of the sign of the component in the direction of $\mathbf{k}\left(\right.$ i.e., $\left.\mathbf{u}_{\mathbf{r}}=\mathrm{u}_{\mathrm{ix}} \mathbf{i}+\mathrm{u}_{\mathrm{iy}} \mathbf{j}-\mathrm{u}_{\mathrm{iz}} \mathbf{k}\right)$. This statement, expressed in everyday language from an educational point of view, is equivalent of saying that the reflection of light occurs as if the photons underwent perfectly elastic collisions with the surface in question. Figure 1 shows a diagram with the notation used for the incident and reflected ray, the normal to the surface and the angles of incidence and reflection.

\subsection{Demonstration of the equivalence of the new and classic statements of the laws of reflection.}

In this section, we show the bidirectional equivalence between the new statement and classic statement:

If the new statement is right, the classic statement must be right as well, both the first part [5] (the angle of incidence equals the angle of reflection),

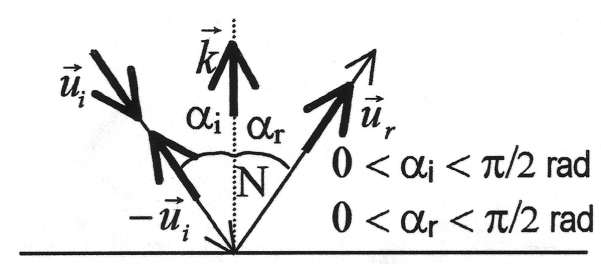

Figure 1: Diagram showing typical reflection 
and the second part [5] (the incident ray, the normal to the surface and the reflected ray, lie all in the same plane), and vice versa.

A) If the new statement is right, the classic statement is right as well

If the new statement is right, we have:

a) The angle $\alpha_{\mathrm{i}}$ between $-\mathbf{u}_{\mathbf{i}}=-\mathrm{u}_{\mathrm{ix}} \mathbf{i}-\mathrm{u}_{\mathrm{iy}} \mathbf{j}-\mathrm{u}_{\mathrm{iz}} \mathbf{k}$ and $\mathbf{k}$, is equal to the angle $\alpha_{\mathrm{r}}$ between $\mathbf{k}$ and $\mathbf{u}_{\mathbf{r}}=\mathrm{u}_{\mathrm{ix}} \mathbf{i}+\mathrm{u}_{\mathrm{iy}} \mathbf{j}-\mathrm{u}_{\mathrm{iz}} \mathbf{k}$, since the three vectors are unit vectors, and the two angles between them are less than $\pi / 2$ radians, the dot product of $-\mathbf{u}_{\mathrm{i}}$ and $\mathbf{k}$ is the same as the dot product of $\mathbf{k}$ and $\mathbf{u}_{\mathrm{r}}$ (demonstration a).

Demonstration a:

Calculate the dot products between $-\mathbf{u}_{\mathrm{i}}$ and $\mathbf{k}$, and between $\mathbf{k}$ and $\mathbf{u}_{\mathrm{r}}$, considering that the angles $\alpha_{\mathrm{i}}$ and $\alpha_{\mathrm{r}}$ are between 0 and $\pi / 2$ radians. Namely, $0<\alpha_{\mathrm{i}}<\pi / 2 \mathrm{rad}$ and $0<\alpha_{\mathrm{r}}<\pi / 2 \mathrm{rad}$ :

$$
\begin{aligned}
\left(-\mathbf{u}_{\mathbf{i}}\right) \cdot \mathbf{k} & =\left(-\mathrm{u}_{\mathrm{ix}} \mathbf{i}-\mathrm{u}_{\mathrm{iy}} \mathbf{j}-\mathrm{u}_{\mathrm{iz}} \mathbf{k}\right) \cdot \mathbf{k} \\
& =-\mathrm{u}_{\mathrm{iz}}=1 \cdot 1 \cdot \cos \alpha_{i} \\
\mathbf{k} \cdot \mathbf{u}_{\mathbf{r}} & =\mathbf{k} \cdot\left(\mathrm{u}_{\mathrm{ix}} \mathbf{i}+\mathrm{u}_{\mathrm{iy}} \mathbf{j}-\mathrm{u}_{\mathrm{iz}} \mathbf{k}\right) \\
& =-\mathrm{u}_{\mathrm{iz}}=1 \cdot 1 \cdot \cos \alpha_{r}
\end{aligned}
$$

then,

$$
\cos \alpha_{r}=\cos \alpha_{i}
$$

and therefore

$$
\alpha_{r}=\alpha_{i}
$$

as was to be shown.

b) The vectors $\mathbf{u}_{\mathrm{i}}=\mathrm{u}_{\mathrm{ix}} \mathbf{i}+\mathrm{u}_{\mathrm{iy}} \mathbf{j}+\mathrm{u}_{\mathrm{iz}} \mathbf{k}$ and $\mathbf{u}_{\mathrm{r}}=\mathrm{u}_{\mathrm{ix}} \mathbf{i}+\mathrm{u}_{\mathrm{iy}} \mathbf{j}-\mathrm{u}_{\mathrm{iz}} \mathbf{k}$ and $\mathbf{k}$ are coplanar, because its triple product is zero (demonstration b).

Demonstration b: k

Calculate the triple product between $\mathbf{u}_{\mathrm{i}}, \mathbf{u}_{\mathrm{r}}$ and

$$
\begin{array}{r}
{\left[\left(\mathbf{u}_{\mathrm{i}}\right) \Lambda \mathbf{u}_{\mathrm{r}}\right] \cdot \mathbf{k}=\left|\begin{array}{ccc}
\mathbf{i} & \mathbf{j} & \mathbf{k} \\
\mathrm{u}_{\mathrm{ix}} & \mathrm{u}_{\mathrm{iy}} & \mathrm{u}_{\mathrm{iz}} \\
\mathrm{u}_{\mathrm{ix}} & \mathrm{u}_{\mathrm{iy}} & -\mathrm{u}_{\mathrm{iz}}
\end{array}\right| \cdot \mathbf{k}} \\
=\left[\left(\mathrm{u}_{\mathrm{ix}}\right) \cdot \mathrm{u}_{\mathrm{iy}}-\left(\mathrm{u}_{\mathrm{iy}}\right) \cdot \mathrm{u}_{\mathrm{ix}}\right] \mathbf{k} \cdot \mathbf{k}=0
\end{array}
$$

This implies that vectors $\mathbf{u}_{\mathrm{i}}, \mathbf{u}_{\mathrm{r}}$ and $\mathbf{k}$ are coplanar.

So it is then proved the first part: if the new law of reflection statement is right, the classic statement is right as well.

B) If the classic statement is right, the news statement is right as well

If now we assume the compliance of the classic formulation of the law of reflection, the unit vectors $\mathbf{u}_{\mathrm{i}}, \mathbf{u}_{\mathrm{r}}$ and $\mathbf{k}$ must be coplanar, and the angle between $-\mathbf{u}_{\mathrm{i}}$ and $\mathbf{k}$ equals the angle between $\mathbf{k}$ and $\mathbf{u}_{\mathrm{r}}$. This requires that both the dot product and the cross product between $-\mathbf{u}_{\mathrm{i}}$ and $\mathbf{k}$ and between $\mathbf{k}$ and $\mathbf{u}_{\mathrm{r}}$ are the same.

This in turn respectively implies that

$$
u_{r z}=-u_{i z}
$$

and

$$
u_{r y}=u_{i y} ; u_{r x}=u_{i x}
$$

and hence

$$
\mathbf{u}_{r}=u_{r x} \mathbf{i}+u_{r y} \mathbf{j}+u_{r z} \mathbf{k}=u_{i x} \mathbf{i}+u_{i y} \mathbf{j}-u_{i z} \mathbf{k}
$$

thus proving true the new statement of the law of reflection (demonstration c).

Demonstration c:

If the dot products between $-\mathbf{u}_{\mathrm{i}}$ and $\mathbf{k}$, and between $\mathbf{k}$ and $\mathbf{u}_{\mathrm{r}}$ are equal:

$\left(-u_{i x} \mathbf{i}-u_{i y} \mathbf{j}-u_{i z} \mathbf{k}\right) \cdot \mathbf{k}=\left(u_{r x} \mathbf{i}+u_{r y} \mathbf{j}+u_{r z} \mathbf{k}\right) \cdot \mathbf{k}$

Then,

$$
u_{r z}=u_{i z}
$$

If the cross products are equal:

$$
\left|\begin{array}{ccc}
\mathbf{i} & \mathbf{j} & \mathbf{k} \\
-\mathrm{u}_{\mathrm{ix}} & -\mathrm{u}_{\mathrm{iy}} & -\mathrm{u}_{\mathrm{iz}} \\
0 & 0 & 1
\end{array}\right|=\left|\begin{array}{ccc}
\mathbf{i} & \mathbf{j} & \mathbf{k} \\
0 & 0 & 1 \\
\mathrm{u}_{\mathrm{rx}} & \mathrm{u}_{\mathrm{ry}} & \mathrm{u}_{\mathrm{rz}}
\end{array}\right|
$$

Then,

$$
-u_{i y} \mathbf{i}+u_{i x} \mathbf{j}=-u_{r y} \mathbf{i}+u_{r x} \mathbf{j}
$$

and,

$$
u_{r x}=u_{i x} ; u_{r y}=u_{i y}
$$

Therefore

$$
\mathbf{u}_{r}=u_{r x} \mathbf{i}+u_{r y} \mathbf{j}+u_{r z} \mathbf{k}=u_{i x} \mathbf{i}+u_{i y} \mathbf{j}-u_{i z} \mathbf{k}
$$

This new statement of the law of reflection is therefore equivalent to the classic statement, as we wanted to prove.

\subsection{Usefulness of this new statement}

Once demonstrated the equivalence of the new and the classic statements of the law of reflection, we can use it to solve optical problems. As an example, this formulation is applied for the resolution of one classic reflection problem proposed to first year students at the university, which resolution without the use of this new formulation requires a considerable number of steps. 
Example: Solving the problem of the three plane mirrors forming a trirectangular trihedron (corner reflector).

Three mutually perpendicular, intersecting plane mirrors are arranged as shown in Figure 2. A light beam is successively reflected on each of them. Demonstrate that the reflected beam at the third mirror is parallel to the first incident beam, but propagating in opposite sense.

A) Solution to this problem using the classic statement:

In Figure 3, angles $\theta, \theta$ and $\theta^{\prime}$ represent the sequential angles of incidence on each of the three reflections. Vectors $\mathbf{N}_{1}, \mathbf{N}_{2}$ and $\mathbf{N}_{3}$ are parallel to the unit vectors $\mathbf{i}, \mathbf{j}$ and $\mathbf{k}$ respectively. The points of incidence in the successive mirrors are $\mathrm{I}_{1}, \mathrm{I}_{2}$ e $\mathrm{I}_{3}$.

We must prove that $\mathbf{u}_{\mathbf{i}}$ and $\mathbf{u}_{\mathbf{r}}$ are antiparallel vectors. First, the steps to the solution using the traditional statement of the laws of reflection are shown. We have tried to explain the reasoning behind the problem resolution in a sequenced and orderly manner, to be as clear as possible for students. In Table 1 we can see the steps to the solution.

B) Solution using the new statement of the law of reflection:

Next is shown the solution to the problem posed using the new statement proposed in this paper, in order to highlight the simplicity and didactic interest of this new statement in opposition to the classical solution.

We take again the problem from Figure 3

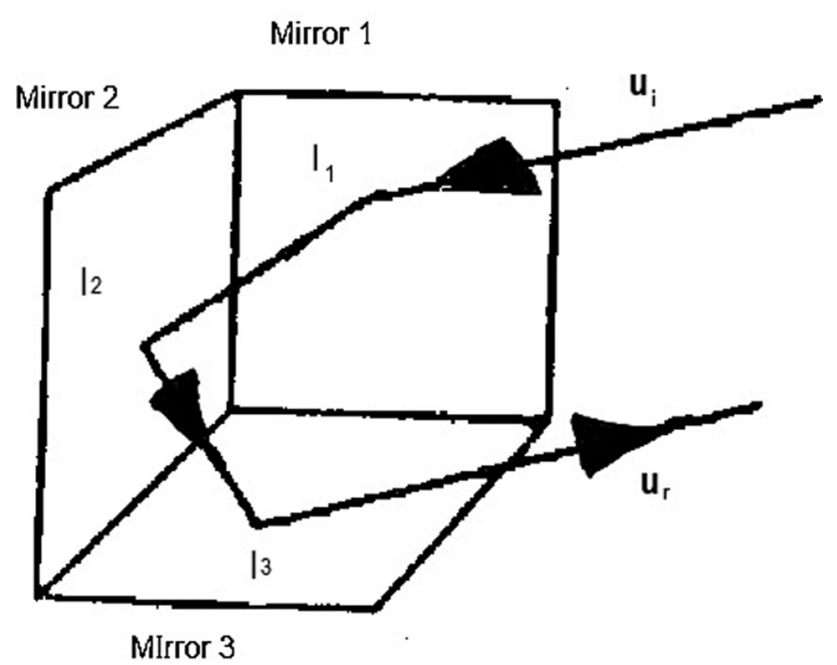

Figure 2: Classic problem of the three plane mirrors forming a trirectangular trihedron

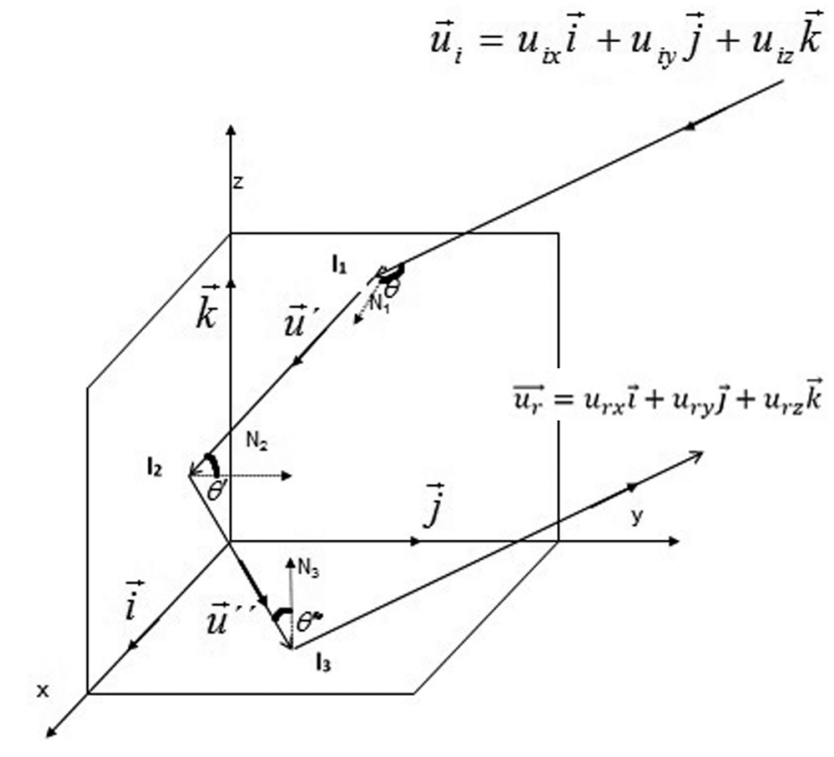

Figure 3: Path of a light beam reflected on three mutually perpendicular, intersecting plane mirrors

The incident ray $\mathbf{u}_{\mathrm{i}}$ can be expressed as

$$
\mathbf{u}_{\mathrm{i}}=\mathrm{u}_{\mathrm{ix}} \mathbf{i}+\mathrm{u}_{\mathrm{iy}} \mathbf{j}+\mathrm{u}_{\mathrm{iz}} \mathbf{k}
$$

Using the new statement of the law of reflection, the beam reflected by the first mirror is,

$$
\mathbf{u}^{\prime}=-\mathrm{u}_{\mathrm{ix}} \mathbf{i}+\mathrm{u}_{\mathrm{iy}} \mathbf{j}+\mathrm{u}_{\mathrm{iz}} \mathbf{k}
$$

Using again our statement of the law of reflection, the reflected beam in the second mirror is,

$$
\mathbf{u}^{\prime \prime}=-\mathrm{u}_{\mathrm{ix}} \mathbf{i}-\mathrm{u}_{\mathrm{iy}} \mathbf{j}+\mathrm{u}_{\mathrm{iz}} \mathbf{k}
$$

The reflected beam on the third mirror will then be

$$
\mathbf{u}_{r}=-\mathrm{u}_{\mathrm{ix}} \mathbf{i}-\mathrm{u}_{\mathrm{iy}} \mathbf{j}-\mathrm{u}_{\mathrm{iz}} \mathbf{k}=-\mathbf{u}_{\mathrm{i}}
$$

As we intended to show, this beam is parallel to the incident beam, but propagating in opposite direction.

\section{Conclusions}

In this work we have presented a new formulation for the law of reflection, and it has been proven its equivalence with the traditional statements appearing in classic books of optics.

The formulation presented in this work allows the interpretation of the reflection phenomenon as analogous to the bounce of an elastic ball on a hard surface. This alternative statement has been of great didactic interest for our students, as they 
Table 1: Steps to the solution of the problem using the classic statement

\begin{tabular}{|c|c|c|}
\hline For the first reflection: & For the second reflection: & For the third reflection: \\
\hline $\begin{array}{l}\text { We can state } \mathbf{u}_{\mathbf{i}}, \mathbf{u}^{\prime}, \text { and } \mathbf{N}_{\mathbf{1}} \text { with their } \\
\text { direction cosines: } \\
\mathbf{u}_{\mathbf{1}}:\left(\boldsymbol{\alpha}_{\mathbf{1}}, \boldsymbol{\beta}_{\mathbf{1}}, \boldsymbol{\gamma}_{\mathbf{1}}\right) \\
\mathbf{u}^{\prime}:\left(\boldsymbol{\alpha}_{\mathbf{2}}, \boldsymbol{\beta}_{\mathbf{2}}, \boldsymbol{\gamma}_{\mathbf{2}}\right) \\
\mathbf{N}_{\mathbf{1}}:(\mathbf{1}, \mathbf{0}, \mathbf{0})\end{array}$ & $\begin{array}{l}\text { Vectors } \mathbf{u}^{\prime}, \mathbf{u} \text { ', and } \mathbf{N}_{\mathbf{2}} \text { can be de- } \\
\text { fined by their direction cosines: } \\
\mathbf{u}^{\prime}:\left(\boldsymbol{\alpha}_{\mathbf{2}}, \boldsymbol{\beta}_{\mathbf{2}}, \boldsymbol{\gamma}_{\mathbf{2}}\right) \\
\mathbf{u}^{\prime \prime}:\left(\boldsymbol{\alpha}_{\mathbf{3}}, \boldsymbol{\beta}_{\mathbf{3}}, \boldsymbol{\gamma}_{\mathbf{3}}\right) \\
\mathbf{N}_{\mathbf{2}}:(\mathbf{0}, \mathbf{1}, \mathbf{0})\end{array}$ & $\begin{array}{l}\text { Analogously, vectors } \mathbf{u} ", \mathbf{u}_{\mathbf{r}}, \text { and } \mathbf{N}_{\mathbf{3}} \\
\text { are defined by their direction cosines: } \\
\mathbf{u}^{\prime \prime}:\left(\boldsymbol{\alpha}_{\mathbf{3}}, \boldsymbol{\beta}_{\mathbf{3}}, \boldsymbol{\gamma}_{\mathbf{3}}\right) \\
\mathbf{u}_{\mathbf{r}}:\left(\boldsymbol{\alpha}_{\mathbf{4}}, \boldsymbol{\beta}_{\mathbf{4}}, \boldsymbol{\gamma}_{\mathbf{4}}\right) \\
\mathbf{N}_{\mathbf{3}}:(\mathbf{0}, \mathbf{0}, \mathbf{1})\end{array}$ \\
\hline $\begin{array}{l}\text { It is verified that: } \\
\mathbf{u}^{\prime}-\mathbf{u}_{\mathbf{1}}=\mathbf{2} \mathbf{N}_{\mathbf{1}} \cos \boldsymbol{\theta}\end{array}$ & $\begin{array}{l}\text { It is verified that: } \\
\mathbf{u}^{\prime \prime}-\mathbf{u}^{\prime}=\mathbf{2} \mathbf{N}_{\mathbf{2}} \cos \boldsymbol{\theta}^{\prime}\end{array}$ & $\begin{array}{l}\text { It is verified that: } \\
\mathbf{u}_{\mathbf{r}}-\mathbf{u}^{\prime \prime}=\mathbf{2} \mathbf{N}_{\mathbf{3}} \cos \boldsymbol{\theta}^{\prime \prime}\end{array}$ \\
\hline $\begin{array}{l}\text { Which can be expressed in their compo- } \\
\text { nents: } \\
\boldsymbol{\alpha}_{2}-\boldsymbol{\alpha}_{1}=\mathbf{2} \cos \boldsymbol{\theta} \\
\boldsymbol{\beta}_{\mathbf{2}}-\boldsymbol{\beta}_{\mathbf{1}}=\mathbf{0} \\
\boldsymbol{\gamma}_{\mathbf{2}}-\boldsymbol{\gamma}_{\mathbf{1}}=\mathbf{0}\end{array}$ & $\begin{array}{l}\text { Which again can be expressed in } \\
\text { their components: } \\
\boldsymbol{\alpha}_{\mathbf{3}}-\boldsymbol{\alpha}_{\mathbf{2}}=\mathbf{0} \\
\boldsymbol{\beta}_{\mathbf{3}}-\boldsymbol{\beta}_{\mathbf{2}}=\mathbf{2} \cos \boldsymbol{\theta}^{\prime} \\
\boldsymbol{\gamma}_{\mathbf{3}}-\boldsymbol{\gamma}_{\mathbf{2}}=\mathbf{0}\end{array}$ & $\begin{array}{l}\text { Expressed in their components: } \\
\boldsymbol{\alpha}_{4}-\boldsymbol{\alpha}_{3}=\mathbf{0} \\
\boldsymbol{\beta}_{4}-\boldsymbol{\beta}_{3}=\mathbf{0} \\
\gamma_{4}-\gamma_{3}=\mathbf{2} \cos \boldsymbol{\theta}^{\prime \prime}\end{array}$ \\
\hline $\begin{array}{l}\theta \text { is the angle between unit vectors }-\mathbf{u}_{\mathbf{i}} \\
\text { and } \mathbf{N}_{\mathbf{1}} \\
\text { then, } \\
\cos \boldsymbol{\theta}=-\boldsymbol{\alpha}_{\mathbf{1}} \cdot \mathbf{1}-\boldsymbol{\beta}_{\mathbf{1}} \cdot \mathbf{0}-\boldsymbol{\gamma}_{\mathbf{1}} \cdot \mathbf{0} \\
\text { and } \cos \boldsymbol{\theta}=-\boldsymbol{\alpha}_{\mathbf{1}} \\
\text { we have then: }\end{array}$ & $\begin{array}{l}\theta^{\prime} \text { is the angle between unit vectors } \\
-\mathbf{u}^{\prime} \text { and } \mathbf{N}_{\mathbf{2}} \\
\text { then, } \\
\cos \boldsymbol{\theta}^{\prime}=-\boldsymbol{\alpha}_{\mathbf{2}} \cdot \mathbf{0}-\boldsymbol{\beta}_{\mathbf{2}} \cdot \mathbf{1}-\boldsymbol{\gamma}_{\mathbf{2}} \cdot \mathbf{0} \\
\text { and } \cos \boldsymbol{\theta}^{\prime}=-\boldsymbol{\beta}_{\mathbf{2}} \\
\text { we have then: }\end{array}$ & $\begin{array}{l}\theta^{\prime \prime} \text { is the angle between unit vectors }-\mathbf{u} " \\
\text { and } \mathbf{N}_{\mathbf{3}} \\
\text { then, } \\
\cos \boldsymbol{\theta}^{\prime \prime}=-\boldsymbol{\alpha}_{\mathbf{3}} \cdot \mathbf{0}-\boldsymbol{\beta}_{\mathbf{3}} \cdot \mathbf{0}-\gamma_{\mathbf{3}} \cdot \mathbf{1} \\
\text { and } \cos \boldsymbol{\theta}^{\prime \prime}=-\gamma_{\mathbf{3}} \\
\text { we have then: }\end{array}$ \\
\hline $\begin{array}{ll}\alpha_{2}-\alpha_{1}=-2 \alpha_{1} & \alpha_{2}=-\alpha_{1} \\
\beta_{2}-\beta_{1}=0 & \beta_{2}=\beta_{1} \\
\gamma_{2}-\gamma_{1}=0 & \gamma_{2}=\gamma_{1}\end{array}$ & $\begin{array}{ll}\alpha_{3}-\alpha_{2}=0 & \alpha_{3}=\alpha_{2} \\
\beta_{3}-\beta_{2}=-2 \beta_{2} & \beta_{3}=-\beta_{2} \\
\gamma_{3}-\gamma_{2}=0 & \gamma_{3}=\gamma_{2} \\
\end{array}$ & $\begin{array}{l}\alpha_{4}-\alpha_{3}=0 \\
\beta_{4}-\beta_{3}=0 \\
\gamma_{4}-\gamma_{3}=-2 \gamma_{3}\end{array}$ \\
\hline \multicolumn{3}{|c|}{ If we take the relations obtained in the three reflections, we have: } \\
\hline \multicolumn{3}{|c|}{$\begin{array}{ll}-\alpha_{1}=\alpha_{2}=\alpha_{3}=\alpha_{4} & \alpha_{1}=-\alpha_{4}\end{array}$} \\
\hline \multicolumn{3}{|c|}{$\begin{array}{ll}\beta_{1}=\beta_{2}=-\beta_{3}=-\beta_{4} & \beta_{1}=-\beta_{4}\end{array}$} \\
\hline \multicolumn{3}{|c|}{$\begin{array}{ll}\gamma_{1}=\gamma_{2}=\gamma_{3}=-\gamma_{4} & \gamma_{1}=-\gamma_{4} \\
\end{array}$} \\
\hline \multicolumn{3}{|c|}{$\begin{array}{l}\text { Therefore, } \mathbf{u}_{\mathbf{1}}=-\mathbf{u}_{\mathbf{r}} \text { that is, the ray reflected on the third mirror and the incident ray on the first mirror are } \\
\text { antiparallel vectors. }\end{array}$} \\
\hline
\end{tabular}

are more used to observe such corpuscular bounce on hard surfaces phenomena that the visualization of reflected waves, which allows them attempting to make easily experimentally testable hypothesis.

This new way of stating the laws of reflection has offered greater educational advantage, both from an interpretation and a meaning point of view of these laws. Thus, certain optical problems can be more clearly and easily solved with this new statement.

In this work we have presented, by way of example, the resolution of the problem of three mutually perpendicular, intersecting plane mirrors forming a trirectangular trihedron (corner reflector). The new developed statement of the law of reflection allows the resolution of traditional problems of geometrical optics, and in some cases the solution is much simpler and more intuitive than that obtained using the traditional statement of the laws of reflection.

\section{Acknowledgments}

The authors wish to thank the Regional Government of Extremadura for its financial support through
Grant GR15102 and GR15009, partially funded by the European Regional Development Fund.

\section{References}

[1] J. Morgan, Introduction to Geometrical and Physical Optics (McGraw-Hill, New York, 1953).

[2] F.W. Sears, M.W. Zemansky, H.D. Young, R.A. Freedman and T.R. Sandin, University Physics (Addison-Wesley, Boston, 1999).

[3] F.A. Jenkins and H.E. White, Fundamentals of $O p$ tics (McGraw-Hill, New York, 1957).

[4] C. Curry, Geometrical Optics (Arnold, London, 1962).

[5] E. Hecht, Optics (Addison-Wesley, Reading, 2000).

[6] R.S. Longhurst, Geometrical and Physical Optics (Orient Longman, London, 1974).

[7] P. Drude, The Theory of Optics (Dover, New York, 1954).

[8] P.R. Bhattacharjee, Eur. J. Phys. 26, 901 (2005).

[9] P.R. Bhattacharjee, Optik 120, 642 (2009).

[10] P.R. Bhattacharjee, Optik 121, 2128 (2010).

[11] P.R. Bhattacharjee, Optik 123, 381 (2012).

[12] E.R. Tkaczyk, Optics Letters 37, 972 (2012).

[13] P.R.Bhattacharjee, Optik 124, 2764 (2013).

[14] P.R. Bhattacharjee, Optik 124, 977 (2013).

[15] P.R. Bhattacharjee, Optik 125, 200 (2014). 